\title{
Étude des interactions entre fourrage et aliment concentré chez le mouton. II. Facteurs de variation de la digestibilité
}

\author{
P Berge 1, JP Dulphy 2 \\ avec la collaboration technique de M Dudilieu, M Jailler, \\ J Jamot, H Bousquet, L L'Hotelier \\ 1 INRA-CRZV de Theix, station de recherches sur la viande, \\ 2 INRA-CRZV de Theix, station de recherches sur la nutrition des herbivores, \\ 63122 Saint-Genès-Champanelle, France
}

(Reçu le 8 avril 1991; accepté le 10 septembre 1991)

\begin{abstract}
Résumé - Deux essais ont été conduits successivement en vue d'étudier les interactions entre les fourrages et les aliments concentrés chez le mouton.

Dans un premier texte ont été présentés les résultats concernant les quantités ingérées d'aliments (variation du taux de substitution du fourrage par le concentré) (Berge et Dulphy, 1985). Ici ont été analysées les variations de la digestibilité de la ration totale. Les effets d'interaction digestive ont été évalués en comparant la digestibilité mesurée de la ration à la digestibilité prévue par le calcul additif (à partir de la digestibilité du foin mesurée lorsque celui-ci est distribué seul et de celle du concentré estimée par régression).

Les facteurs de variation étudiés ont été la nature et la proportion de l'aliment concentré dans la ration, la nature du fourrage et le niveau d'alimentation des animaux.

Sept aliments concentrés ont été utilisés. Ils étaient respectivement constitués principalement de pellicules de soja, son de blé, graines de céréales, pulpe de betterave, un mélange lupin-maïs (essai I), orge et à nouveau de la pulpe de betterave (essai II). Chaque combinaison foin-concentré a été distribuée successivement ad libitum puis en quantité limitée aux besoins d'entretien à 12 moutons répartis en 4 groupes de 3 animaux avec respectivement $0,30,60$ ou $90 \%$ d'aliment concentré dans la ration. On a mesuré la digestibilité de la matière organique, des matières azotées totales, de la cellulose brute et de l'amidon. De plus, une partie des régimes étudiés a été distribuée à des moutons fistulisés du rumen pour mesurer l'activité cellulolytique, le $\mathrm{pH}$ et la teneur en $\mathrm{NH}_{3}$ et en acides gras volatils du liquide du rumen.

Dans 4 cas seulement (pellicules de soja et céréales dans les rations consommées ad libitum et orge, 2 fois, dans les rations distribuées en quantité limitée), la digestibilité de la ration n'a pas augmenté de façon linéaire avec la proportion de concentré, ce qui traduit l'existence d'une interaction digestive. La diminution du niveau alimentaire, consécutive à la restriction des quantités ingérées d'aliments, a eu pour effet d'accroître la digestibilité de la ration, mais de façon inégale selon l'aliment concentré.

Sur l'ensemble des rations distribuées contenant 30,60 et $90 \%$ de concentré $(n=193)$, la valeur moyenne des effets d'interaction digestive a été pratiquement nulle, effets positifs et négatifs s'annulant. En valeur absolue, ces effets ont une valeur moyenne de 1,8 point de digestibilité et ont été plus élevés avec les concentrés à base de pellicules de soja, de céréales et d'orge. Ils ont augmenté avec la teneur en amidon de la ration et baissé avec la teneur en matières azotées de celle-ci.

Les interactions digestives ont essentiellement porté sur la digestibilité de la cellulose brute, c'est-àdire, vraisemblablement sur les constituants pariétaux de la ration. Mais les mécanismes mis en jeu
\end{abstract}


ont été différents selon les cas. Les interactions observées avec les rations contenant les concentrés à base de graines de céréales ont été expliquées par la baisse de l'activité cellulolytique dans le rumen, consécutive à l'ingestion de quantités croissantes et importantes d'amidon. Dans le cas du concentré à base de pellicules de soja, l'interaction a été due avant tout à une forte augmentation des quantités ingérées ad libitum lorsque la proportion de concentré a augmenté. Le très probable accroissement de la vitesse de transit du contenu digestif qui en est résulté, et la réduction de l'activité cellulolytique dans le rumen, peuvent expliquer la baisse importante de la digestibilité des pellicules de soja.

Nous concluons qu'il est possible de minimiser les phénomènes d'interaction digestive et leur conséquence, les effets associatifs de digestibilité, en limitant les quantités ingérées par les animaux à un niveau voisin de la satisfaction de leurs besoins d'entretien, et en distribuant des rations à teneur faible ou modérée en amidon et à teneur suffisamment élevée en matières azotées.

\section{foin / concentré / digestibllité / interaction digestive / ovin}

Summary - Study of Interactions between forages and concentrates in sheep. II. Digestibility variation factors. Interactions between forages and concentrates were studied in sheep in the course of 2 successive trials. Results concerning intake (variations in forage/concentrate substitution rates) have been presented in a previous paper (Berge and Dulphy, 1985). In the present study variations in digestibility have been examined for different associations between a forage and a concentrate. Effects of digestive interaction have been evaluated by comparing measured digestibility of the diet to predicted digestibility (prediction based on hay digestibility measured when fed alone and concentrate digestibility calculated by regression) (fig 1). The following variation factors were examined: type and proportion of concentrate, type of forage and level of animal feeding.

Seven concentrates based on soyabean hulls, wheat-bran, cereals, beet pulp and lupin (trial I), and barley and beet pulp (trial II) were used. Each diet combining one hay and one concentrate was given ad libitum and then at maintenance level to 12 sheep with $0,30,60$ and $90 \%$ concentrate in the diet ( 3 animals per diet). Digestibility was measured for organic matter, crude protein, crude fiber and starch. Moreover, some of these diets were offered to fistulated sheep to measure cellulolytic activity, VFA, $\mathrm{pH}$ and level of $\mathrm{NH}_{3}$ in the rumen (table I).

In 4 situations only (soybean hulls and cereals in diets fed ad libitum; and barley twice, in diets fed at maintenance), digestibility with the same hay/concentrate combination did not increase linearly with proportion of concentrate thus showing digestive interactions (cf figs 2 and 3; table III). At decrease in feeding level by the restriction of allocated feeds, resulted in an increase in diet digestibility, more or less with the type of concentrate (tables IV, V).

For the whole diets containing 30,60 and $90 \%$ concentrate $(n=193)$ the average value of the effects of digestive interactions was practically nil, positive and negative values counterbalancing. The average absolute value of these effects was 1.8 , and higher for concentrates based on soyabean hulls, cereals and barley. It increased with starch level and decreased with crude protein level of the diets.

Digestive interactions mainly concern crude fiber, ie cell wall content of the feeds. However, the mechanims involved the latter differed from one situation to another. With diets containing cereals, interactions can be explained by a decrease in cellulolytic activity in the rumen due to the intake of a significant and increasing quantity of starch (table VII). With diets containing soyabean hulls, the interaction can be explained above all by a large increase in ad libitum intake. In case a probable increase in digesta flow, and a decrease in cellulolytic activity, can jointly explain an important decrease in soybean hull digestibility.

It is concuded that it is possible to minimise digestive interactions and so the decrease in diet digestibility by restricting feeds offered at maintenance level and by preparing diets with low starch content and sufficiently high crude protein content.

\section{hay / concentrate / digestibility / digestive interaction / sheep}




\section{INTRODUCTION}

L'addition d'aliment concentré aux fourrages distribués ad libitum à des ruminants modifie d'une part, les quantités de fourrage ingérées, d'autre part la digestibilité des constituants de la ration. Ces phénomènes constituent ce qu'il est convenu d'appeler les interactions entre aliments. Les interactions concernant l'ingestion ont fait l'objet d'une présentation antźrieure (Berge et Dulphy, 1985).

Les interactions concernant la digestibilité se traduisent, en général, par une valeur de la digestibilité de la ration, mesurée sur l'animal, différente de celle calculée additivement en faisant la somme pondérée des digestibilités du fourrage et du concentré mesurées séparément (Demarquilly et al, in INRA, 1978) : on parle alors d'interaction digestive négative ou positive selon que la digestibilité mesurée est respectivement inférieure ou supérieure à la valeur attendue. En d'autres termes, les interactions digestives se manifestent par une variation de la digestibilité de la ration non proportionnelle à la variation de la part pondérale du concentré dans cette ration (fig 1). La digestibilité du fourrage est obtenue le plus souvent en le distribuant seul aux animaux, alors que celle du concentré, plus difficile à mesurer (Giger ef Sauvant, 1983), est généralement extraite de tables.

Les principaux facteurs de variation connus des effets d'interaction digestive, pour un animal donné, sont :

- la proportion de concentré dans la ration (Demarquilly et al, in INRA 1978; Dulphy et al, 1983; Guérin et Dulphy, 1984);

- la nature du fourrage : les interactions digestives négatives augmentent avec la teneur en cellulose brute du fourrage (Demarquilly et al, in INRA, 1978) et sont importantes dans le cas des pailles (Dulphy et al, 1983). Plus précisément, elles sont positives en présence de faibles quantités de concentré et deviennent fortement négatives lorsque l'apport de concentré augmente;

- la nature du concentré : les interactions digestives négatives sont plus importantes lorsque le concentré est à base de graines de céréales que lorsqu'il s'agit de concentrés moins riches en amidon comme la pulpe de betterave et le son de blé (Muller et Béranger, 1979; Vérité et Dulphy, 1981). Par contre, elles varient en sens inverse de la teneur en matières azotées totales de la ration (Dulphy et al, 1982; Guérin et Dulphy, 1984);

- le niveau alimentaire des animaux (Joanning et al, 1981; Moe, 1981; Robinson et al, 1987; Edionwe et Owen, 1989).

Deux essais ont donc été réalisés pour préciser les effets :

- de la nature du fourrage et de celle du concentré,

- des proportions respectives de ces aliments dans la ration et

- du niveau d'alimentation sur la digestibilité de la ration totale chez le mouton en vue d'évaluer les interactions digestives et leurs facteurs de variation.

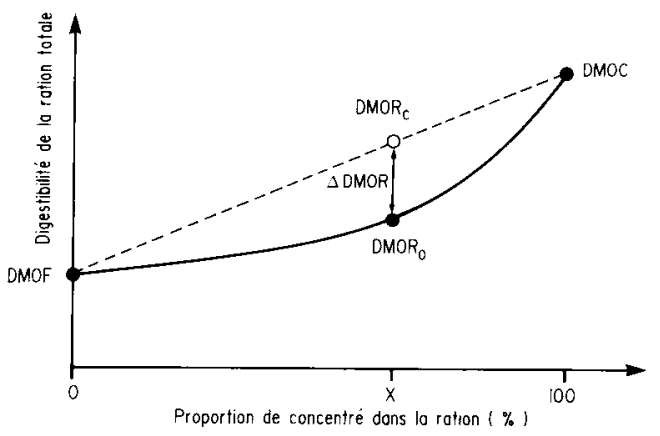

Fig 1. Conséquence des interactions digestives sur la prévision de la digestibilité des rations mixtes. $D M O R_{0}=$ digestibilité observée de la ration; $D M O R_{c}=$ digestibilité calculée de la ration; $D M O F=$ digestibilité du fourrage; $D M O C=$ digestibilité du concentré; $\triangle D M O R=$ interaction digestive. 


\section{MATÉRIEL ET MÉTHODES}

Les conditions dans lesquelles se sont déroulés les 2 essais, ainsi que les caractéristiques des aliments utilisés et les analyses chimiques réalises ont déjà été présentées par Berge et Dulphy (1985).

\section{Schéma expérimental}

Douze moutons adultes castrés (en moyenne $62 \pm 6 \mathrm{~kg}$ de poids vif dans l'essai I; $66,5 \pm 5 \mathrm{~kg}$ dans l'essai II) ont reçu successivement 7 aliments concentrés différents ( 5 dans l'essai I, 2 dans l'essai II) associés à différents foins, de sorte que le concentré représentait $0,30,60$ et $90 \%$ respectivement de la matière sèche (MS) totale de la ration, à raison de 3 moutons par niveau de concentré pour chaque combinaison d'un fourrage et d'un concentré donnés. Chaque combinaison fourrage/concentré a été distribuée ad libitum sur la base d'un taux de refus du fourrage de $10 \%$ pendant 21 j (15 j d'adaptation et $6 \mathrm{j}$ de mesure) et, immédiatement après et dans les mêmes conditions, en quantité limitée aux besoins d'entretien du mouton, estimés à $23 \mathrm{~g}$ de matière organique digestible/kg PV0.75 (INRA, 1978).

Dans le premier essai, les 5 concentrés (constitués principalement de pellicules de soja, de son de blé, de graines de céréales, de pulpe de betterave déshydratée, et d'un mélange de lupin et de maïs, respectivement) ont chacun été associés à l'un des 5 foins ( 1 foin de fléole et 4 foins de prairie naturelle). Les foins, bien que différents, avaient des ingestibilités assez voisines $(64,7 \pm 6,6 \mathrm{~g} \mathrm{MS} / \mathrm{kg} P V 0,75)$.

Dans le second essai, les 2 concentrés (orge et pulpe de betterave déshydratée) ont chacun été associés dans les mêmes conditions que dans l'essai 1 , à 3 foins différents $(2$ foins de prairie naturelle, l'un issu d'une coupe tardive de premier cycle (FG), lautre d'une coupe précoce de deuxième cycle $(R G)$ et un foin de luzerne (FL).

Par ailleurs, 4 moutons castrés adultes et fistulisés * ont été utilisés pour mesurer des paramètres de la digestion dans le rumen (activité cellulolytique, $\mathrm{pH}$, teneur en azote ammoniacal et en acides gras volatils du liquide du rumen). Une partie seulement des traitements réalisés sur les moutons en mesure de digestibilité a pu être reproduite sur les moutons fistulisés (tableau I).

\section{Analyse statistique des résultats}

Les valeurs individuelles de la digestibilité de la matière organique (DMO) ont été analysées par régression afin d'établir des équation reliant la $D M O$ de la ration totale (DMOR) au pourcentage de matière organique du régime sous forme de concentré $\left(C_{m o}\right)$ selon le modèle

Tableau I. Régimes étudiés sur les moutons fistulisés.

\begin{tabular}{|c|c|c|c|c|c|c|c|c|c|}
\hline \multirow{2}{*}{\multicolumn{2}{|c|}{$\begin{array}{l}\text { Niveau d'alimentation } \\
\text { Niveau de concentré (\%) }\end{array}$}} & \multicolumn{4}{|c|}{ Quantité limitée } & \multicolumn{4}{|c|}{ ad libitum } \\
\hline & & 0 & 30 & 60 & 90 & 0 & 30 & 60 & 90 \\
\hline Concentré & Foin & & & & & & & & \\
\hline $\begin{array}{l}\text { Essai I } \\
\text { Pellicules de soja }\end{array}$ & Prairie naturelle & * & * & * & * & * & * & * & * \\
\hline $\begin{array}{l}\text { Essai II } \\
\text { Orge } \\
\text { Pulpe de betterave }\end{array}$ & $\begin{array}{l}\text { Pr nat (RG) } \\
\text { Pr nat (RG) }\end{array}$ & & & $\star$ & & * & * & * & * \\
\hline
\end{tabular}

\footnotetext{
- Les animaux ont été opérés par J Lefaivre que nous tenons à remercier
} 
$D M O R=a+b C+c C^{2}($ ff fig 1). Cette démarche avait pour objectif de :

- déceler l'existence d'éventuelles interactions digestives en testant la signification (au seuil de $5 \%$ de probabilité) du terme quadratique de l'équation (test de curvilinéarité). En effet, il a été posé comme hypothèse que la non-linéarité de la relation entre $D M O R$ et $C$ traduit la variation non proportionnelle de DMOR en réponse à l'augmentation du pourcentage de concentré, et par conséquent, la non additivité des quantités de constituants digestibles des ingrédients de la ration;

- calculer la $D M O$ du concentré (DMOC) en extrapolant à $C=100 \%$ l'équation de régression linéaire ou curvilineaire selon qu'il y avait interaction digestive ou non :

- calculer additivement la DMOR à partir de la DMO du foin (mesurée) et de la DMO du concentré (calculée par régression pour $C=$ 100), et par là, l'interaction digestive représentée par la différence $\triangle D M O R$ entre la digestibilité mesurée et la digestibilité calculée de la ration.

De plus, l'effet du niveau d'ingestion a été étudié par régression multiple :

- soit en testant le niveau alimentaire (NA en multiples des besoins d'entretien sur la base de la matière organique digestible ingérée) : DMOR $=a+b C_{m o}+c N A$

- soit en testant directement les quantités totales de $M S$ ingérées ( $Q I$ en $\mathrm{g} / \mathrm{kg} P \mathrm{PV}^{0.75}$ ): $D M O R=a+b C_{m o}+c Q I$

Enfin, les valeurs de la digestibilité $D M O R$ d'une part et de l'effet associatif de digestibilité $\triangle D M O R$ d'autre part ont été soumises à une analyse de variance et covariance suivant le modèle élaboré par Seebeck (1973), afin de tester la signification des effets principaux des facteurs (nature du concentré, nature du foin, niveau d'alimentation) et des covariables (pourcentage de concentrés, teneurs en MAT, cellulose brute et amidon de la ration), ainsi que celles des interactions de premier ordre entre les facteurs.

Les valeurs individuelles de la digestibilité de la cellulose brute (DCBR) ont été analysées par régression pour établir des équations reliant la $D C B R$ de la ration totale au pourcentage de concentré $\left(C_{C B}=\right.$ proportion de cellulose brute du régime dans le concęntré) selon le modèle $D C B R=a+b C_{C B}+c C_{C B}^{2}$ (fig 6).

\section{RÉSULTATS}

\section{Variations de la digestibilité de la matière organique de la ration (DMOR)}

L'analyse de la variance globale de DMOR a montré que les 3 sources de variation étudiées (nature et proportion du concentré, niveau alimentaire) ont eu un effet hautement significatif $(P<0,001)$ de même que l'interaction nature du concentré $x$ proportion de concentré $(P<0,001)$ et, dans une moindre mesure, les interactions de la nature du concentré et de sa proportion avec le niveau alimentaire $(P<0,05)$. $\AA$ titre d'exemple le résultat de 2 analyses est donné dans le tableau II.

\section{Effet de la proportion de concentré}

Les variations de la DMOR en fonction de la proportion de concentré dans la ration sont représentées sur les figures 2 et 3 . Le meilleur ajustement a été obtenu par régression linéaire dans presque tous les cas. Le coefficient du terme quadratique a cependant été significatif dans le cas des rations à base de pellicules de soja $(P S)$ et de céréales (CC) distribuées à volonté dans l'essai I. II l'a été également dans l'essai II avec les rations contenant l'orge $(O)$ associée aux foins $F G$ et $F L$, mais distribuées en quantités limitée (tableau III).

Dans les 4 cas de rations, il y a donc eu interaction digestive, mais cette dernière ne s'est pas toujours manifestée dans le même sens. Elle a été négative dans le cas du concentré à base de céréales et positive dans les 3 autres cas.

\section{Effet du niveau d'alimentation}

Les diverses rations distribuées ad libitum ont été consommées en quantité très va- 
Tableau II. Effet des principales sources de variation sur la digestibilité de la matière organique des rations (valeurs de F).

\begin{tabular}{lcc}
\hline & Essail & Essai II \\
\hline Concentré & 34 & 9 \\
Foin & - & 58 \\
Niveau d'alimentation & 119 & 118 \\
Proportion de concentré (\%C) & 441 & 110 \\
Concentré x \%C & 35 & - \\
Variance expliquée (\%) & 88 & 91 \\
\hline
\end{tabular}

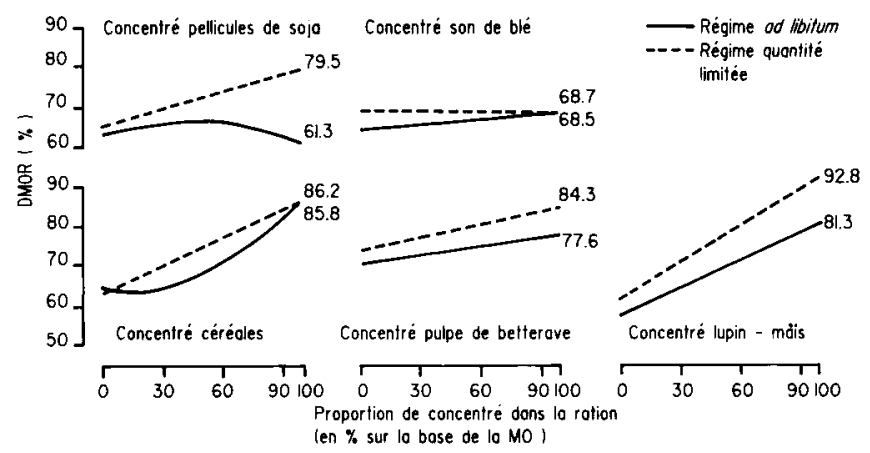

Flg 2. Variation de la digestibilité de la ration (DMOR) en fonction de la proportion de concentré et du niveau d'alimentation (Essai I).

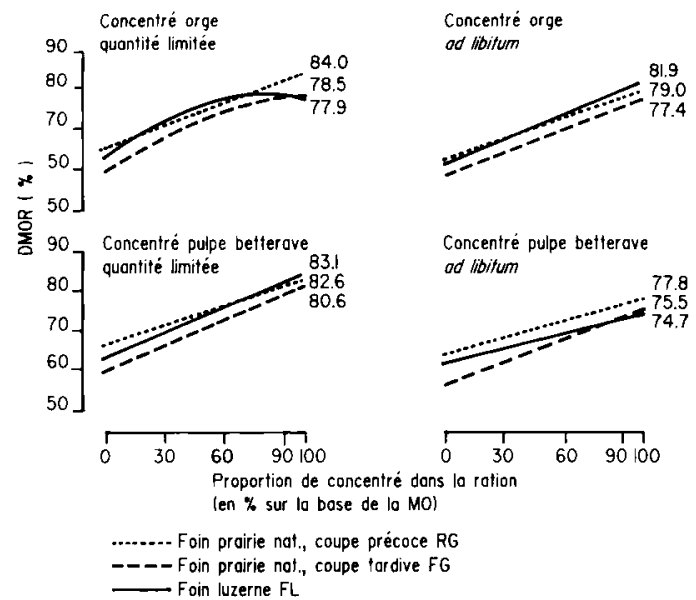

Fig 3. Variation de la digestibilité de la ration (DMOR) en fonction de la proportion de concentré, de la nature du foin associé et du niveau d'alimentation (Essai II). riable, non seulement d'un concentré à l'autre, mais aussi d'une proportion de concentré à l'autre. II en a résulté une diftérence maximale de niveau alimentaire de 0,3 à 1,5 selon les essais, soit en moyenne $0,8 \pm 0,4$ fois les besoins d'entretien des moutons (tableau IV).

Les effets liés aux variables $C^{2}$ mo et $N A^{2}$ ont été faibles et donc négligés.

Le coefficient attaché à la variable $N A$ dans les équations de régression multiple $D M O R=a+b C+c N A$ (tableau V) montre qu'une augmentation d'une unité du niveau alimentaire a entraîné une baisse de la digestibilité de la ration de 2,8 à 7,7 points selon le type de concentré. L'effet du niveau alimentaire a été plus fort avec les concentrés à base de pellicules de soja et 
Tableau III. Équations de régression reliant la digestibilité de la ratio (DMOR, en \%) à la proportion de concentré $\left(C_{M O}\right.$ en $\%$ ) de la forme $D M O R=a+b C_{M O}+C C 2_{M O}(2)$.

\begin{tabular}{|c|c|c|c|c|c|c|c|c|}
\hline Essai & $\begin{array}{l}i \text { Niveau Co } \\
\text { d'alimentation }\end{array}$ & oncentré & Foin & $a$ & $\begin{array}{l}b \\
(1)\end{array}$ & $\begin{array}{l}c \\
(1)\end{array}$ & Syx & $r$ \\
\hline 1 & limité & $\begin{array}{l}\text { PS } \\
\text { SB } \\
\text { CC } \\
\text { PB } \\
\text { LM } \\
\text { PS } \\
\text { SB } \\
\text { CC } \\
\text { PB } \\
\text { LM }\end{array}$ & & $\begin{array}{l}63,9 \\
64,0 \\
64,4 \\
70,3 \\
57,9 \\
65,3 \\
68,8 \\
63,0 \\
73,7 \\
61,8\end{array}$ & $\begin{array}{c}0,144^{*} \\
0,045^{\star} \\
-0,086 \mathrm{NS} \\
0,073^{* \star *} \\
0,234^{* * *} \\
0,142^{*} \\
-0,0001 \mathrm{NS} \\
0,232^{* \star *} \\
0,107^{* * *} \\
0,309^{* * *}\end{array}$ & $\begin{array}{l}-0,0017^{*} \\
\text { NS } \\
0,0030^{*} \\
\text { NS } \\
\text { NS } \\
\text { NS } \\
\text { NS } \\
\text { NS } \\
\text { NS } \\
\text { NS }\end{array}$ & $\begin{array}{l}2,0 \\
1,8 \\
2,6 \\
1,2 \\
1,3 \\
2,5 \\
1,8 \\
1,7 \\
1,1 \\
1,9\end{array}$ & $\begin{array}{l}0,658^{\star} \\
0,649^{*} \\
0,934^{* * *} \\
0,914^{* * *} \\
0,989^{* * *} \\
0,900^{* * *} \\
0,020 \mathrm{NS} \\
0,975^{* * *} \\
0,961^{* * *} \\
0,987^{* * *}\end{array}$ \\
\hline II & ad libitum & $P$ & $\begin{array}{l}R G \\
F G \\
F L \\
R G \\
F G \\
F L \\
R G \\
F G \\
F L \\
R G \\
F G \\
F L\end{array}$ & $\begin{array}{l}62,8 \\
57,9 \\
61,7 \\
64,7 \\
56,1 \\
62,0 \\
65,6 \\
59,9 \\
63,5 \\
66,0 \\
59,7 \\
63,1\end{array}$ & 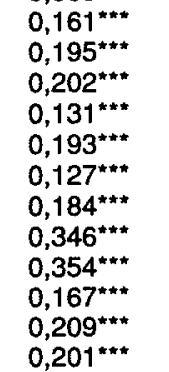 & $\begin{array}{l}\text { NS } \\
\text { NS } \\
\text { NS } \\
\text { NS } \\
\text { NS } \\
\text { NS } \\
\text { NS } \\
-0,0016^{* *} \\
-0,0021^{* *} \\
\text { NS } \\
\text { NS } \\
\text { NS }\end{array}$ & $\begin{array}{l}2,0 \\
1,7 \\
2,6 \\
1,5 \\
1,9 \\
1,4 \\
1,7 \\
1,0 \\
1,4 \\
1,3 \\
2,2 \\
1,2\end{array}$ & $\begin{array}{l}0,934^{* * *} \\
0,967^{* \star *} \\
0,936^{* * *} \\
0,955^{* * *} \\
0,964^{* * *} \\
0,950^{* * *} \\
0,969^{* * *} \\
0,993^{* * *} \\
0,978^{* * *} \\
0,976^{* * *} \\
0,960^{* * *} \\
0,986^{* * *}\end{array}$ \\
\hline
\end{tabular}

(1) Significatif au seuil de probabilité $5 \%\left({ }^{*}\right), 1 \%\left({ }^{* *}\right), 0,1 \%\left(f^{* *}\right)$ ou non significatif (NS).

(2) $C_{M O}$ est la proportion de concentré sur la base de la matière organique.

Tableau IV. Quantités ingérées (QI, g MS $/ \mathrm{kg} P V 0.75$ ) et niveau alimentaire (NA, en multiple des besoins d'entretien*) selon le type de ration distribuée ad libitum et la proportion de concentré (moyennes sur 3 moutons).

\begin{tabular}{|c|c|c|c|c|c|c|c|c|c|}
\hline \multicolumn{4}{|c|}{ Type de ration } & \multicolumn{4}{|c|}{ Proportion de concentré } & \multirow{2}{*}{\multicolumn{2}{|c|}{$90 \%$}} \\
\hline Concentré & Foin & $0 \%$ & & 30 & & 60 & & & \\
\hline & & QI & NA & QI & NA & QI & NA & Ql & NA \\
\hline \multirow[t]{5}{*}{ Essai I } & PS & 54,6 & 1,4 & 67,4 & 1,9 & 73,6 & 2,0 & 95,4 & 2,5 \\
\hline & SB & 63,3 & 1,7 & 70,4 & 1,9 & 68,2 & 1,8 & 48,2 & 1,3 \\
\hline & $\mathrm{CC}$ & 70,7 & 1,9 & 76,0 & 2,0 & 67,6 & 1,9 & 50,4 & 1,7 \\
\hline & PB & 72,3 & 2,1 & 81,9 & 2,4 & 82,7 & 2,5 & 78,6 & 2,4 \\
\hline & $\mathrm{LM}$ & 63,6 & 1,5 & 68,1 & 1,8 & 78,0 & 2,3 & 72,8 & 2,4 \\
\hline \multirow[t]{6}{*}{ Essai II } & $O(R G)$ & 68,0 & 1,6 & 74,6 & 2,1 & $\mathbf{5 7 , 7}$ & 1,7 & 50,2 & 1,6 \\
\hline & $(\mathrm{FG})$ & 61,1 & 1,5 & 68,0 & 1,8 & 51,3 & 1,4 & 46,0 & 1,5 \\
\hline & $(\mathrm{FL})$ & 73,7 & 1,8 & 84,3 & 2,2 & 56,5 & 1,7 & 38,9 & 1,3 \\
\hline & $P(R G)$ & 61,8 & 1,6 & 69,4 & 2,0 & 77,5 & 2,3 & 64,9 & 2,0 \\
\hline & (FG) & 55,1 & 1,3 & 60,8 & 1,5 & 59,3 & 1,6 & 61,7 & 1,8 \\
\hline & (FL) & 71,4 & 1,7 & 76,7 & 2,0 & 68,4 & 1,9 & 55,5 & 1,6 \\
\hline
\end{tabular}

" Estimés à $23 \mathrm{~g}$ MO digestible/kg PV0,75 (INRA, 1978). 
Tableau V. Équations de régression reliant la digestibilité de la matière organique de la ration (DMOR) au pourcentage de concentré $\left(C_{M O}\right)$ et, soit au niveau alimentaire $N A$ (DMOR $=\mathrm{a}+b C_{M O}+$ cNA), soit aux quantités de MS ingérées QI $(D M O R=a+b c+c Q I)$.

\begin{tabular}{|c|c|c|c|c|c|c|c|}
\hline Concentré & Foin & $a$ & $b^{\prime}$ & $c^{\prime}$ & Syx & $R$ & $n$ \\
\hline \multicolumn{8}{|l|}{$N A$} \\
\hline $\begin{array}{r}\text { Essai I PS } \\
\text { SB } \\
\text { CC } \\
\text { PB } \\
\text { LM }\end{array}$ & & $\begin{array}{l}74,0 \\
72,5 \\
68,8 \\
78,0 \\
69,7\end{array}$ & $\begin{array}{l}0,110^{* * *} \\
0,011 \mathrm{NS} \\
0,192^{* * *} \\
0,098^{* * *} \\
0,316^{* * *}\end{array}$ & $\begin{array}{l}-6,84^{* * *} \\
-4,12^{* * *} \\
-3,92^{* * *} \\
-3,67^{* * *} \\
-7,66^{* * *}\end{array}$ & $\begin{array}{l}3,2 \\
1,9 \\
3,1 \\
1,5 \\
1,8\end{array}$ & $\begin{array}{l}0,814 \\
0,669 \\
0,906 \\
0,938 \\
0,985\end{array}$ & $\begin{array}{l}23 \\
24 \\
24 \\
24 \\
23\end{array}$ \\
\hline \\
\hline $\begin{array}{r}\text { Essai II } \\
\begin{array}{r}O \\
P\end{array}\end{array}$ & $\begin{array}{l}R G \\
F G \\
F L \\
R G \\
F G \\
F L\end{array}$ & $\begin{array}{l}69,7 \\
66,6 \\
68,6 \\
69,4 \\
64,8 \\
68,7\end{array}$ & $\begin{array}{l}0,173^{* * *} \\
0,199^{* * *} \\
0,172^{* * *} \\
0,155^{* * *} \\
0,221^{* * *} \\
0,167^{* * *}\end{array}$ & $\begin{array}{l}-4,07^{* * *} \\
-5,40^{* * *} \\
-3,18^{*} \\
-2,83^{* * *} \\
-5,94^{* * *} \\
-4,46^{* * *}\end{array}$ & $\begin{array}{l}2,0 \\
2,1 \\
2,4 \\
1,6 \\
2,4 \\
2,2\end{array}$ & $\begin{array}{l}0,948 \\
0,958 \\
0,939 \\
0,957 \\
0,949 \\
0,943\end{array}$ & $\begin{array}{l}24 \\
22 \\
24 \\
23 \\
24 \\
24\end{array}$ \\
\hline \multicolumn{8}{|l|}{ QI } \\
\hline $\begin{array}{r}\text { Essai I PS } \\
\text { SB } \\
\text { CC } \\
\text { PB } \\
\text { LM }\end{array}$ & & $\begin{array}{l}73,8 \\
72,6 \\
70,3 \\
77,8 \\
71,6\end{array}$ & $\begin{array}{l}0,103^{* * *} \\
0,009 \mathrm{NS} \\
0,173^{* * *} \\
0,091^{* * *} \\
0,272^{* * *}\end{array}$ & $\begin{array}{l}-0,179^{\star \star \star *} \\
-0,114^{\star \star \star} \\
-0,126^{\star \star *} \\
-0,102^{\star \star *} \\
-0,206^{\star \star * *}\end{array}$ & $\begin{array}{l}2,8 \\
1,7 \\
2,9 \\
1,4 \\
1,7\end{array}$ & $\begin{array}{l}0,859 \\
0,730 \\
0,919 \\
0,943 \\
0,987\end{array}$ & $\begin{array}{l}23 \\
24 \\
24 \\
24 \\
23\end{array}$ \\
\hline \multicolumn{8}{|l|}{$Q I$} \\
\hline Essai II 0 & $\begin{array}{l}R G \\
F G \\
F L \\
R G \\
F G \\
F L\end{array}$ & $\begin{array}{l}70,3 \\
67,6 \\
69,7 \\
69,8 \\
66,4 \\
69,0\end{array}$ & $\begin{array}{l}0,158^{* * *} \\
0,176^{* * *} \\
0,155^{* * *} \\
0,146^{* * *} \\
0,198^{* * *} \\
0,152^{* * *}\end{array}$ & 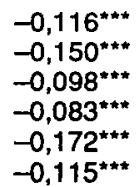 & $\begin{array}{l}2,0 \\
1,9 \\
2,2 \\
1,5 \\
2,2 \\
2,1\end{array}$ & $\begin{array}{l}0,953 \\
0,965 \\
0,948 \\
0,961 \\
0,960 \\
0,946\end{array}$ & $\begin{array}{l}24 \\
22 \\
24 \\
23 \\
24 \\
24\end{array}$ \\
\hline
\end{tabular}

(1) NS, ", "*, *** : cf tableau III.

de lupin-maïs (baisse d'environ 7 points) et le plus faible avec les concentrés à base de pulpe de betterave (baisse d'environ 4 points en moyenne).

La diminution de la DMOR a aussi été d'autant plus importante que la proportion de concentré était plus élevée, en moyenne $-2,2 ;-3,9 ;-5,9$ et $-5,8$ points pour une unité de niveau alimentaire en plus aux niveaux $0,30,60$ et $90 \%$ de concentré respectivement.
Par rapport à la variable $N A$, la variable $Q I$ a eu un effet légèrement plus élevé (tableau V). Le coefficient attaché à cette variable montre qu'une augmentation de $10 \mathrm{~g}$ de $\mathrm{MS} / \mathrm{kg} \mathrm{P}^{0,75}$ des quantités totales ingérées a entraîné une baisse de la digestibilité de la ration de 0,83 à 2,06 points selon le type de concentré. Les autres conclusions concernant l'effet de la variable $Q I$ sont les mêmes que celles concernant l'effet de la variable $N A$. 
Contribution des différents constituants à la variation de la teneur en matière organique digestible de la ration

La variation de la digestibilité de la ration totale est la résultante des variations respectives de la digestibilité de ses constituants chimiques parmi lesquels nous avons retenu les matières azotées totales (MAT), la cellulose brute (CB) et l'amidon. Cependant, pour tenir compte des variations importantes de la composition chimique de la ration dues à la nature et la proportion des aliments étudiés, il convient de raisonner également en termes de teneur de la ration en constituants digestibles qui associe les 2 paramètres de la digestibilité du constituant et de sa concentration dans la ration.

La digestibilité des matières azotées de la ration (DMAR) dépend essentiellement de la teneur en MAT de la ration. Cette dernière variable a expliqué plus de $95 \%$ de la variabilité de la $D M A R$ dans les 2 essais.

De plus, mis à part les rations contenant des aliments à teneur élevée en MAT (concentré lupin-maïs, essai l, et les foins $R G$ et $F L$, essai II), la teneur en MAT de la ration totale a été pratiquement constante (fig 4). Ceci explique, en partie, que les variations de la teneur en matières azotées digestibles (MAD) de la ration aient été relativement modestes et qu'elles n'aient contribué que pour une faible part aux variations de la matière organique digestible (MOD) de la ration (fig 5).

Au contraire, la teneur en cellulose brute digestible $(C B D)$ des rations a été beaucoup plus variable (fig 5). En effet, la teneur en $C B$ de la ration a diminué de 6 à 23 points (en moyenne 15 points) entre les proportions 0 et $90 \%$ de concentré si l'on excepte le cas des pellicules de soja (augmentation de 4 points) (fig 4). Ensuite, la digestibilité de la cellulose brute (DCB) a varié de façon très différente selon le type

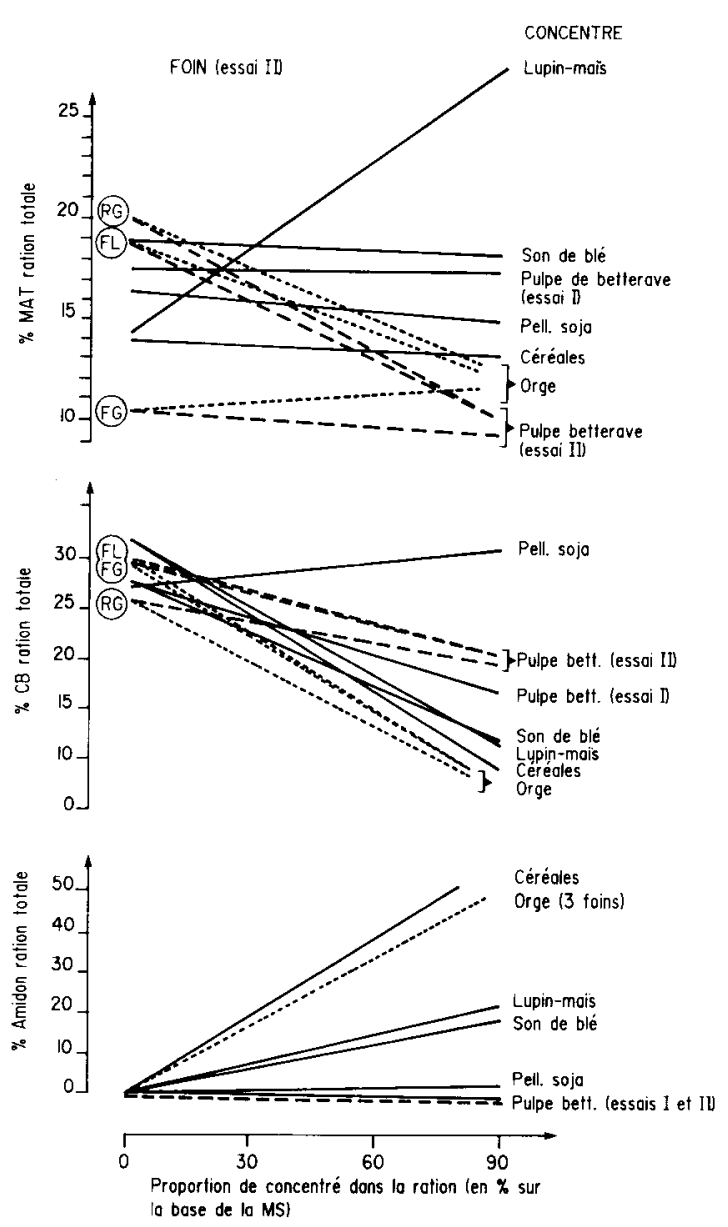

Fig 4. Variation de la composition chimique de la ration totale en fonction de la proportion de concentré.

de ration, d'abord en raison des écarts importants de $D C B$ entre concentrés, mais aussi à cause de phénomènes d'interaction digestive (fig $6 a, b$ ).

Ainsi, alors qu'au niveau de l'entretien, la $D C B$ des rations contenant les pellicules de soja a augmenté avec la proportion de concentré, en régime ad libitum celle-ci a diminué dès $30 \%$ de concentré (base MS). 
ESSAI I
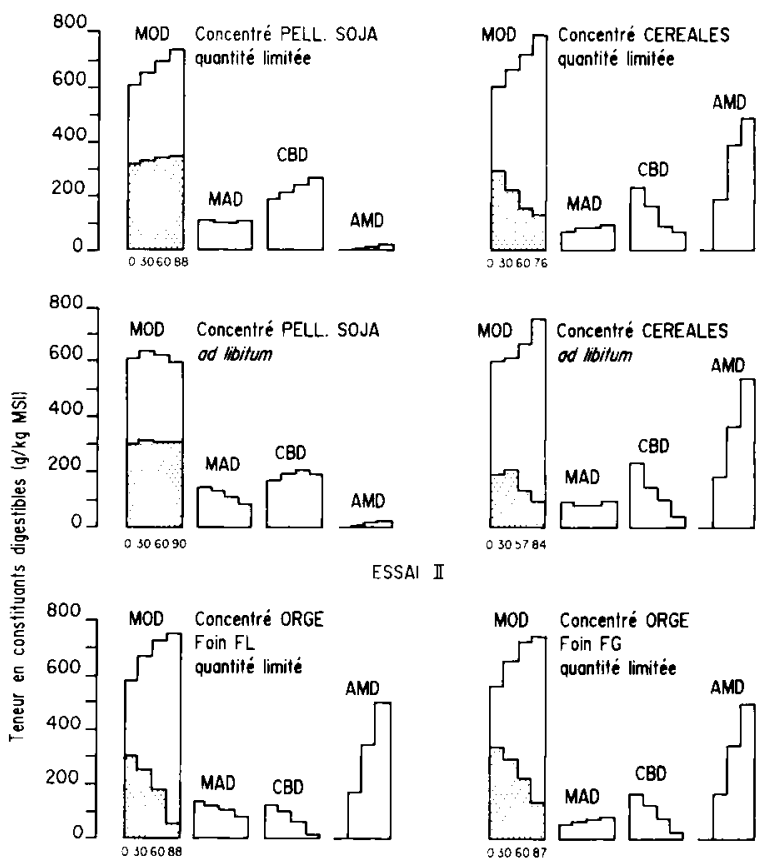

ESSAI $\mathbb{I}$
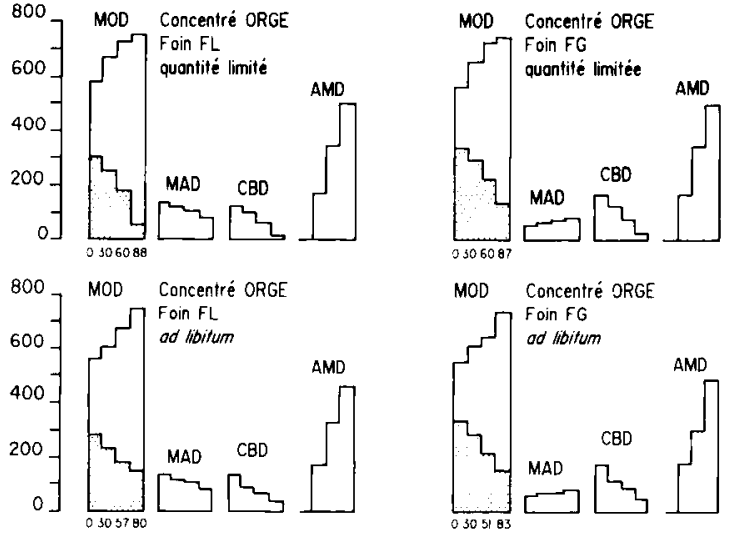

Proportion moyenne de concentré dans lo ration ( \% )

Fig 5. Variation des teneurs en matière organique digestible (MOD), matières azotées digestibles (MAD), cellulose brute digestible (CBD) et amidon digestible (AMD) de la ration en fonction de la proportion de concentré dans la ration (en grisé, fraction de la MOD non déterminée par l'analyse chimique).

Cette baisse de la $D C B$ est à mettre au compte de l'effet d'un accroissement des quantités ingérées (tableau IV) puisque dans les rations contenant le concentré lupin-maïs, on a également observé des évolutions contraires de la $D C B$ selon qu'elles étaient distribuées en quantité limitée ou ad libitum. Une interaction néga- tive similaire sur la $D C B$ s'est manifestée dans le cas de l'orge associée aux foins $F G$ et $F L$ de l'essai II à l'entretien, du fait d'une diminution très importante $(-20$ points) de la $D C B$ entre les niveaux 60 et $90 \%$ de concentré sur la base de la MS.

La digestibilité de l'amidon des concentrés étudiés a toujours été très élevée 

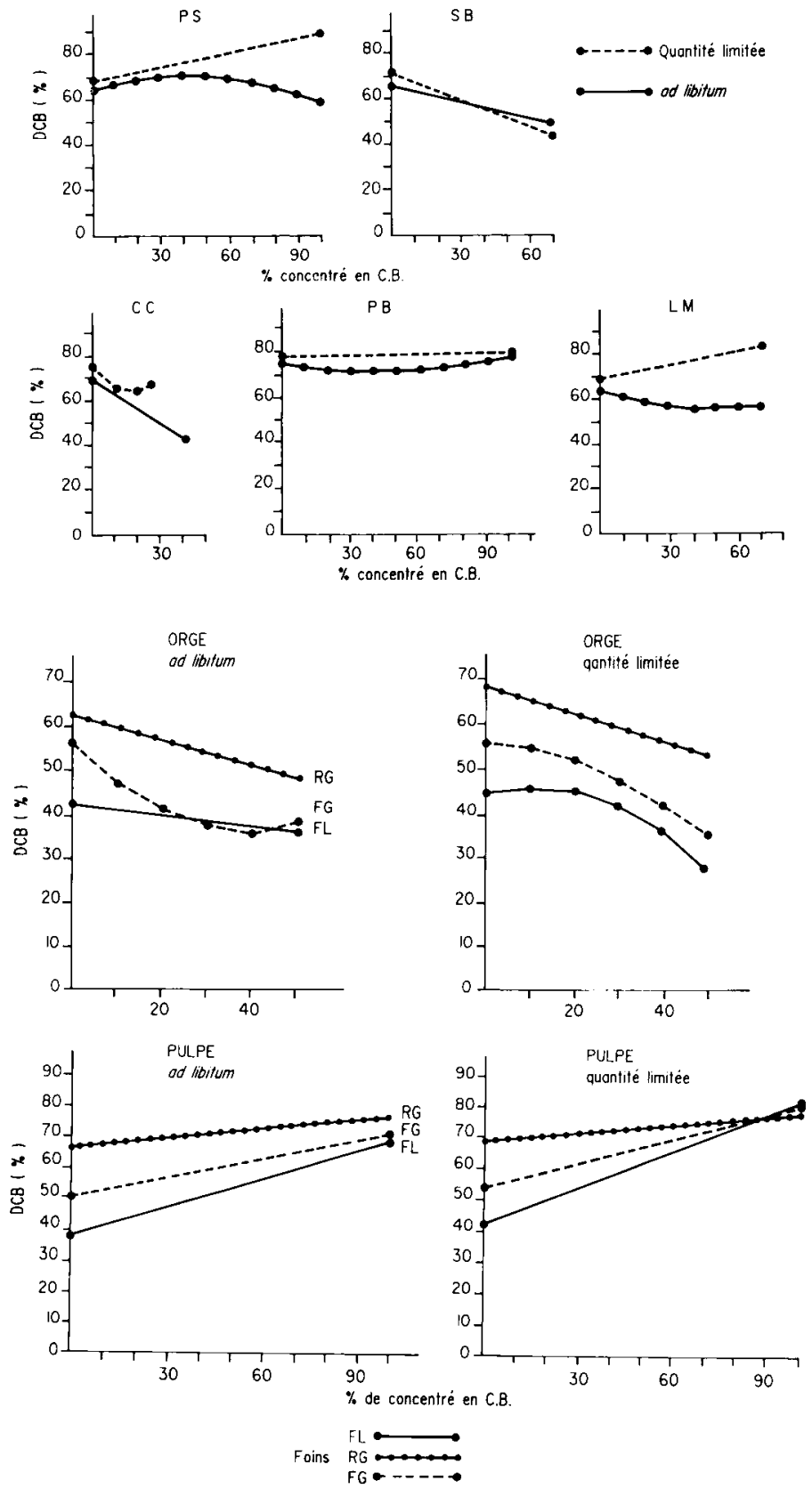

Fig 6. a. Essai I : variation de la digestibilité de la cellulose brute (DCB) de la ration en fonction de la proportion de concentré dans la ration (sur la base de la cellulose brute). b. Essai II : variation de la DCB de la ration en fonction de la proposition de concentré dans la ration (sur la base de la cellulose brute). 
( $\geq 94 \%$ dans l'essai I, $\geq 98 \%$ dans l'essai II). Elle a cependant légèrement et significativement augmenté avec la proportion de concentré (en moyenne 3 points d'augmentation entre 30 et $90 \%$ de concentré) et significativement diminué avec le niveau d'alimentation (en moyenne 1 point de digestibilité en moins par unité de niveau alimentaire en plus). L'augmentation de la teneur en amidon de la ration s'est donc répercutée totalement sur la DMOR et sur la teneur en $M O D$ de la ration.

\section{Mesure des effets associatifs de digestibilité}

Sur l'ensemble des 2 essais, on a calculé la valeur des effets associatifs de digestibilité représentés, pour chacune des mesures de digestibilité individuelles, par l'écart $(\triangle D M O R)$ entre la digestibilité de la ration mesurée (DMOR mes) et celle calculée $\left(D M O R_{c a l d}\right)$ à partir des digestibilités respectives de ses constituants : $\triangle D M O R$ $=D M O R_{\text {mes }}-D M O R_{\text {calc }}$

La moyenne globale de ces effets associatifs est pratiquement nulle $(-0,016 \pm$ 2,4 points; $n=193$ mesures de $D M O R$ ). Leur variation a été indépendante de la proportion de concentré (en moyenne $-0,2$ $+0,1$ et $+0,1$ aux niveaux 30,60 et $90 \%$ de concentré respectivement, différences non significatives, $P>0,05$ ). Par contre, elle a dépendu significativement du niveau d'alimentation $(+0,33$ en régime quantité limitée à l'entretien, et $-0,37$ en régime ad libitum) en raison des interactions observées avec les régimes ad libitum dans l'essai et avec les régimes quantité limitée dans l'essai II (tableau VI).

Le fait que les interactions digestives se soient manifestées tantôt positivement, tantôt négativement ne permet pas, au travers de l'étude des moyennes algébriques, d'apprécier l'amplitude (ou intensité) de ces interactions. En effet, étant de signes contraires selon que l'interaction a été positive ou négative, les valeurs des effets associatifs s'annulent dans le calcul des moyennes, ce qui contribue à masquer la variation de l'intensité (ou valeur absolue) de ces effet associatifs.

Pris en valeur absolue, les effets associatifs (valeur moyenne $1,8 \pm 1,6$ point) n'ont pas dépendu de la proportion de concentré $(P>0,05)$. Par contre, les effets du niveau d'alimentation et de la nature du concentré ont été très significatifs $(P<$ $0,001)$ : ils ont été les plus élevés avec le

Tableau VI. Analyse de variance des interactions digestives (en valeur algébrique).

\begin{tabular}{|c|c|c|c|c|c|c|c|}
\hline \multirow[t]{2}{*}{ Essai concerné } & \multicolumn{4}{|c|}{ Effects principaux des facteurs } & \multicolumn{3}{|c|}{ Interactions fer ordre } \\
\hline & (NC) & (NF) & $\begin{array}{c}\text { concentré } \\
\text { (PC) }\end{array}$ & $\begin{array}{l}\text { d'alimentation } \\
\text { (NA) }\end{array}$ & $n+N C \times N A$ & $N F \times N A$ & $\mathrm{NC} \times \mathrm{NF}$ \\
\hline \multirow{2}{*}{$\begin{array}{l}\text { Essai I } \\
(n=88) \\
\text { Essai II } \\
(n=105)\end{array}$} & $* * *$ & - & NS & NS & $m$ & - & - \\
\hline & * & NS & NS & **** & $* \star *$ & ** & NS \\
\hline $\begin{array}{l}\text { Essais I+ II } \\
(n=193)\end{array}$ & $* * *$ & - & NS & $\star$ & *** & - & - \\
\hline
\end{tabular}

Effet significatif au seuil de probabilité de $0,1 \%\left({ }^{* * *}\right), 1 \%\left(^{* *}\right), 5 \%\left(^{*}\right)$ ou non significatif (NS). 
concentré céréales (en moyenne 3,6 points) et les pellicules de soja (2,3 points) de l'essai I, et l'orge (2,2 points) de l'essai II. Ils ont été les plus faibles avec les pulpes de betterave $(1,1$ point en moyenne sur les 2 essais).

Le regroupement de tous les résultats a permis d'établir la relation suivante entre l'intensité (ou valeur absolue) des effets associatifs et les paramètres de la composition chimique de la ration : $|\triangle D M O R|=2,04-$ $0,044 M A T+0,029 A M I( \pm 1,55 ; r=0,327 ; n$ = 193) où les teneurs en MAT et amidon (AMI) sont exprimées en \% de la MS.

Cette équation montre que l'intensité des effets associatifs de digestibilité augmente avec la teneur en amidon de la ration, donc du concentré. Elle indique aussi que la teneur en MAT tend à exercer un effet opposé à celui de l'amidon.

La teneur en $C B$ de la ration n'apparaît pas dans l'équation en raison de sa corrélation élevée avec la teneur en amidon ( $r=-$ 0,$823 ; n=193$ ). En fait, elle a le même effet, quoique moins important et non significatif, que la teneur en MAT de la ration.

\section{Caractéristiques du jus de rumen}

Les valeurs moyennes des principaux paramètres de la digestion dans le rumen ont été regroupées dans le tableau VII. Une partie de ces résultats a été déjà présentée (Berge et Dulphy, 1985).

\section{Activité cellulolytique}

Dans les 2 essais, l'activité cellulolytique du jus de rumen a diminué à chaque augmentation de la proportion de concentré, mais n'a pas été beaucoup modifiée par la limitation des quantités ingérées.

Dans l'essai II, à même proportion de concentré, on a pu constater qu'elle était inférieure de 4-10 points avec l'orge com- parée à la pulpe après un séjour de 48-72 $h$ des sachets dans le rumen, temps moyen que requièrent les constituants pariétaux pour être dégradés à ce niveau.

\section{Acidité (pH)}

Le pH moyen a toujours diminué quand la proportion de concentré a augmenté. L'effet du niveau d'alimentation a, par contre, été très faible. Au cours de la journée, les variations du $\mathrm{pH}$ ont été plus importantes avec l'orge qu'avec la pulpe (essai II: avec l'orge, le $\mathrm{pH}$ a diminué jusqu'à 5,4 en restant le plus souvent inférieur à 6,0 dans les $2-4 \mathrm{~h}$ suivant le repas, alors qu'avec la pulpe il est pratiquement toujours resté supérieur à 6,0 ).

\section{Azote ammoniacal $\left(\mathrm{N}-\mathrm{NH}_{3}\right)$}

Les teneurs en $\mathrm{N}-\mathrm{NH}_{3}$ du jus de rumen ont été presque toujours relativement élevées dans les $8 \mathrm{~h}$ suivant le repas. Néanmoins, elles ont été particulièrement faibles dans l'essai II quand la ration contenait plus de $60 \%$ de pulpe (teneurs moyennes inférieures à $50 \mathrm{mg} / \mathrm{l}$ de jus) et dans une moindre mesure l'orge. La limitation des quantités ingérées a eu, dans ces cas-là, pour effet d'augmenter très fortement ces teneurs (de 70 à $180 \%$ en variation relative).

\section{Acides gras volatils}

La teneur en acides gras volatils totaux (AGVT) du jus de rumen a augmenté avec la proportion de concentré, sauf dans le cas de l'orge une fois dépassés les $30 \%$ de concentré (essai II).

L'orientation des fermentations n'a étémodifiée de façon marquée par la proportion de concentré que dans le cas de l'orge, avec une diminution de la proportion des acides $\mathrm{C}_{2}$ (acétique) au profit des 
P Berge, JP Dulphy

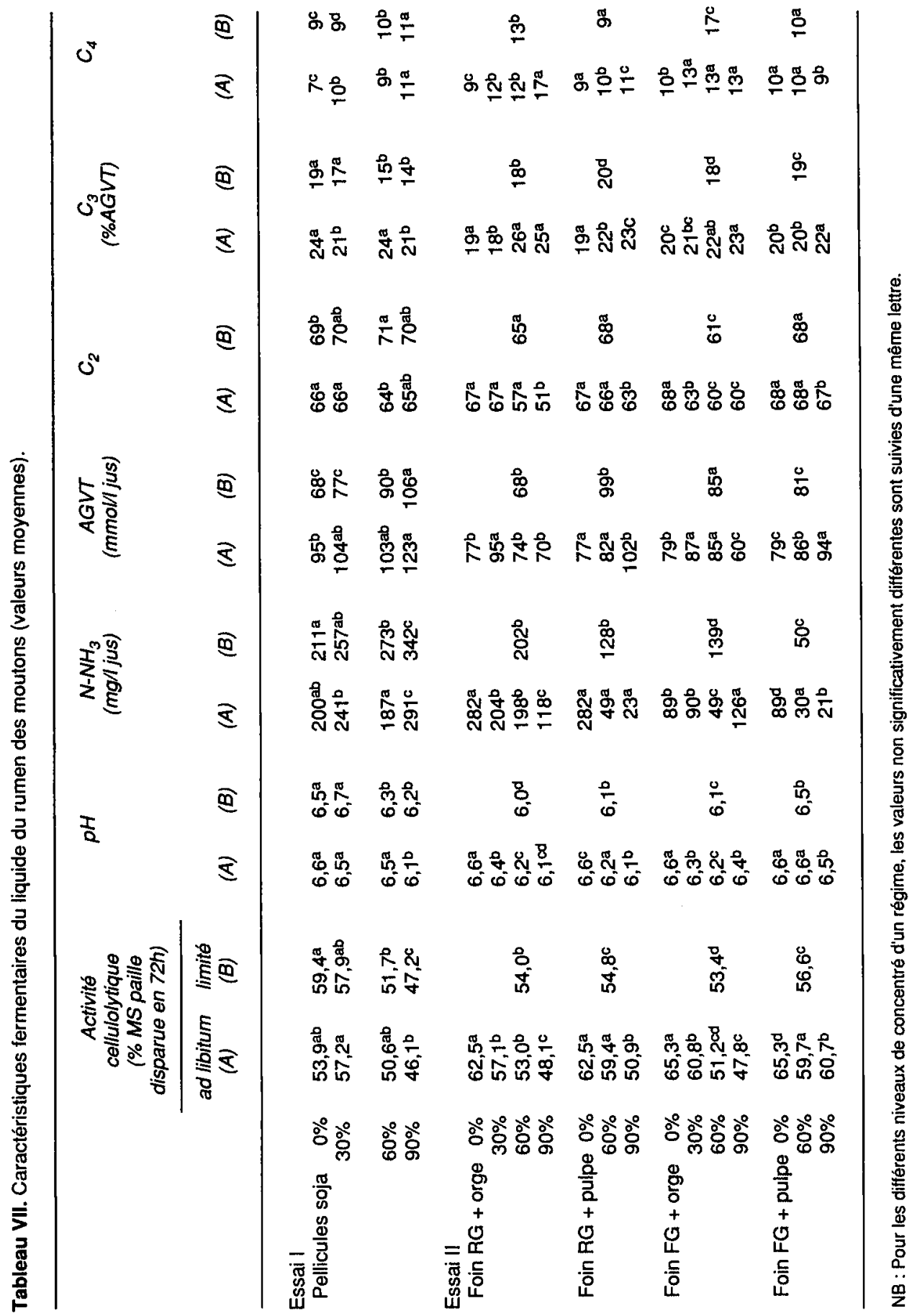


acides en $\mathrm{C}_{3}$ (propionique) et $\mathrm{C}_{4}$ (butyrique) (respectivement $-15,+17$ et +8 points entre 0 et $90 \%$ de concentré). La même tendance, mais plus discrète, a été observée avec la pulpe du même essai.

Le seul effet de la limitation des quantités ingérées a été de réduire la teneur en AGVT de $20 \%$ en moyenne quand la ration contenait des pellicules de soja de l'essai I. Dans l'essai II, cet effet n'a pas été observé.

\section{DISCUSSION}

Des observations réalisées dans les 2 essais, il est apparu que les variations de la digestibilité de la ration totale ne sont pas toujours proportionnelles à l'accroissement du pourcentage de concentré dans la ration. Elles confirment donc que, lorsqu'on associe fourrages et aliments concentrés, peuvent se manifester des phénomènes d'interaction digestive, tantôt positive (cas des pellicules de soja de l'essai l, et de l'orge de l'essai II), tantôt négative (cas du concentré céréales de l'essai l). Des phénomènes semblables ont été rapportés auparavant par Blaxter et Wilson (1963), Blaxter et Wainman (1964), Joanning et al (1981) et Mould et al (1983b).

L'apparition des interactions, tant au niveau de l'ingestion que de la digestibilité de la ration, peut s'expliquer par certaines modifications induites par l'ingestion des concentrés dans les processus digestifs. Ces modifications ont été présentées et discutées précédemment par Berge et Dulphy (1985) et Michalet-Doreau et Sauvant (1989).

Dans nos essais, les interactions digestives ne se sont pas manifestées au niveau de la digestibilité de l'amidon (ou de la teneur en amidon digestible de la ration), toujours restée très élevée. De même, la digestibilité des MAT ne semble pas devoir être mise en cause, et ce, d'autant moins que la teneur en MAD de la ration a peu varié dans les régimes où l'interaction digestive s'est manifestée, n'expliquant de ce fait qu'une faible part des variations de la teneur en MOD de la ration (fig 5). On peut aussi raisonnablement admettre que, comme l'amidon, les glucides solubles des aliments sont presque complètement digérés. En somme, si l'on fait abstraction de la fraction non déterminée par l'analyse chimique $(10-50 \%$ de la $M O D$ totale), on peut conclure que les interactions digestives ont porté essentiellement sur la digestibilité de la cellulose brute, fraction représentant les constituants pariétaux des aliments, comme cela a été suggéré auparavant par Mould et al (1983b). Par contre, elles n'ont pas porté sur la digestibilité de l'amidon comme cela avait été constaté par Joanning et al (1981).

L'interaction observée avec les pellicules de soja (essai I) est due, pour une bonne part, à l'augmentation importante des quantités ingérées entre 0 et $90 \%$ de concentré. Cette augmentation a provoqué une baisse marquée de la digestibilité de la cellulose brute, surtout au-delà de $60 \%$ de concentré, alors qu'en régime quantité limitée, celle-ci n'avait cessé d'augmenter (fig 6a). En effet, l'activité cellulolytique a diminué lorsque la proportion du concentré a augmenté. Ce phénomène est probablement dû, au moins partiellement, à l'acidification du contenu du rumen (Osbourn et al, 1970; Lamb et Eadie, 1979; Mould et al, 1983a; Shriver et al, 1984; Berge et Dulphy, 1985; Ørskov, 1986; Giger et al, 1988). Mais le fait que la baisse de l'activité cellulolytique et $\mathrm{du} \mathrm{pH}$ dans le rumen n'ait pas été plus forte en régime ad libitum qu'en quantité limitée, permet de penser que la diminution de la digestibilité des constituants pariétaux dans le rumen n'a pas été compensée par un temps de séjour plus long des aliments à ce niveau. À l'effet dépressif observé sur l'activité cellu- 
lolytique a donc dû s'ajouter celui de l'accélération de la vitesse de transit du contenu digestif au niveau du rumen conformément à la description faite par Balch et Campling (1962) et par Najar et al (1988).

L'interaction observée dans le même essai avec le concentré céréales n'est, au contraire, pas liée à des variations de quantités ingérées. C'est avec ce concentré que le niveau alimentaire a le moins varié en régime ad libitum. On peut émettre l'hypothèse d'une valeur anormalement élevée de la digestibilité du foin distribué seul. Cependant, le sens de cette interaction (négative) est conforme au schéma classique proposé pour un en décrire l'effet au niveau de la digestibilité de la ration.

Les interactions observées avec les régimes distribués enquantité limitée dans l'essai II, sont également délicates à interpréter. Elles sont bien associées à une forte interaction digestive au niveau de la cellulose brute au-delà de $30 \%$ d'orge dans la ration alors que dans les rations contenant de la pulpe, la digestibilité de ce constituant ne cessait d'augmenter (fig 6b). Dans l'ensemble, les valeurs d'activité cellulolytique et de $\mathrm{pH}$ du jus de rumen ont été plus faibles avec l'orge qu'avec la pulpe, et l'ingestion de l'orge, même en régime quantité limitée, a entraîné dans le rumen une chute du $\mathrm{pH}$ après le repas plus forte que celle de la pulpe (Giger et al, 1988). Néanmois, faute d'un nombre suffisant de mesures des paramètres de la digestion dans le rumen pour les rations contenant l'orge, il n'est pas possible d'apporter d'explication satisfaisante à l'effet propre du niveau d'alimentation. Mais un phénomène similaire d'interaction digestive positive chez le mouton nourri à l'entretien a été observé au cours d'essais menés ultérieurement par MichaletDoreau (données non publiées) avec du corn-feed associé en proportion variable avec le même foin de luzerne que celui de l'essai II.
Dans ces essais, les temps de séjour des aliments dans le tube digestif, et en particulier dans le rumen, n'ont pas été mesurés. Ils auraient probablement éclairé les résultats concernant l'activité cellulolytique et ceux concernant la digestibilité des rations. En effet, la digestion des parois s'effectue essentiellement dans le rumen et dépend principalement de 3 paramètres : la résistance propre de ces parois à la mastication et aux attaques microbiennes, l'activité cellulolytique du contenu du rumen et le temps de séjour de ces parois dans le rumen. Si ce temps diminue, la digestibilité des parois diminue.

Plusieurs facteurs importants jouent un rôle sur le temps de séjour des parois végétales dans le rumen : la nature du fourrage (Mambrini, 1990), la nature du concentré (Poncet et al, 1987a), le pourcentage de concentré (Poncet et al, $1987 \mathrm{~b}$ ) et le niveau d'alimentation (Poncet et al, 1987a, b; Najar et al, 1988; Peyraud et al, 1988).

Ainsi, lorsque la proportion de concentré augmente, le temps de séjour du fourrage dans le rumen s'accroit; lorsque le niveau d'alimentation est diminué, ce temps de séjour s'accroît également. Dans le premier cas, la baisse de l'activité cellulolytique l'emporte et il y a baisse de la digestibilité des parois. Dans le second cas, l'accroissement du temps de séjour entraîne en général une augmentation de la digestibilité des parois.

Une partie des interactions générées au niveau du rumen pourraient être atténuées au niveau du gros intestin. Cependant, les digesta n'y séjournent que quelques h (4-8 selon Mambrini (1990) et il est probable que cette durée ne permette guère de modifier des différences notables apparues dans le rumen.

L'intérêt de la comparaison des résultats obtenus sur des moutons fistulés aux 2 niveaux d'alimentation est limité du fait 
des faibles niveaux d'ingestion qu'ils ont réalisés en régime ad libitum (niveau alimentaire moyen 1,45 contre 1,76 pour les moutons en digestibilité). La teneur en $\mathrm{N}$ $\mathrm{NH}_{3}$ a pu être un facteur limitant de l'activité microbienne dans le rumen notamment dans le cas de régimes contenant la pulpe pour lesquels les valeurs moyennes ne dépassaient pas le seuil de 50 à $100 \mathrm{mg} / \mathrm{l}$ de jus (INRA, 1978). Mais cette hypothèse ne semble pas devoir être retenue dans le cas des régimes contenant l'orge de l'essai II. Tout au plus, le développement de termentations butyriques (tableau VII) indique une perturbation des fermentations du même type que celles classiquement observées avec les rations à base de concentrés riches en amidon (Eadie et al, 1970; Lyle et al, 1981).

Les effets associatifs de digestibilité, tels qu'ils ont été mesurés dans ce travail, n'ont pas varié significativement avec le pourcentage de concentré dans la ration. Globalement, ils ont été négligeables, c'est-à-dire que sur le total des 193 mesures, leur moyenne algébrique a été nulle. Autrement dit, sur l'ensemble des mesures, il n'y a ni sur- ni sous-estimation de la digestibilité des rations par le calcul additif. La valeur absolue moyenne de ces effets associatifs est faible, ce qui montre que, bien que les interactions digestives existent réellement, elles n'ont pas eu d'effet important sur les résultats d'ensemble. Néanmoins, l'analyse des résultats confirme que les facteurs favorables à l'apparition d'effets associatifs importants sont, au niveau de la ration, une teneur élevée en amidon (apportée par des aliments comme les graines de céréales; Hynd, 1984), une teneur forte en cellulose brute (ou constituants pariétaux apportés entre autres par les fourrages), et une teneur faible en MAT.

II faut cependant préciser que les écarts $(\triangle D M O R)$ que nous avons assimilés aux effets associatifs de digestibilité ne sont pas en réalité uniquement la conséquence des interactions digestives mais aussi celle de l'imprécision sur la mesure de la digestibilité du fourrage seul, et de celle de la ration totale (ou variabilité due à l'animal), ainsi que de l'imprécision sur le calcul de la digestibilité du concentré (dans le cas de nos essais, erreur sur l'estimation par régression).

Ces résultats ne sont donc pas en accord avec ceux de Demarquilly (in INRA, 1978) et Guerin et Dulphy (1984) qui ont trouvé, chez le mouton alimenté ad libitum, des effets associatifs toujours négatifs (moyennes de $-1,8$ et de -2 à -4 points respectivement) en utilisant les coefficients de digestibilité des tables INRA (1978). Ces coefficients sont des valeurs moyennes issues d'une compilation des données de la bibliographie. Elles surestiment la digestibilité des concentrés parce que, dans l'ensemble, elles ont été obtenues sur des animaux nourris à l'entretien. Ceci est évident lorsqu'on compare les digestibilités des concentrés obtenues dans nos essais aux 2 niveaux d'alimentation (figs 2 et 3 ). La surestimation de la digestibilité du concentré aura entraîné une surestimation systématique de la digestibilité de la ration totale calculée additivement, et d'autant plus importante que la proportion de concentré était la plus élevée puisque la digestibilité du fourrage était, elle, mesurée et donc sujette à la même erreur que le concentré. La preuve en est apportée par les résultats portés dans le tableau VIII. Les effets associatifs ainsi mesurés en régime ad libitum auraient été surestimés de 3 points du seul fait de l'absence de correction pour l'effet du niveau alimentaire sur la digestibilité du concentré. Néanmoins, Dulphy et al (1982), Guérin et Dulphy (1984) et divers autres auteurs cités par Hynd (1984) observent, comme dans nos essais, que les effets associatifs 
Tableau VIII. Comparaison des résultats du calcul des interactions digestives ( $\triangle D M O R$, en points en $\%$ ) selon que la digestibilité du concentré est prise dans les tables (a) ou qu'elle est calculée par régression aux deux niveaux d'alimentation (b).

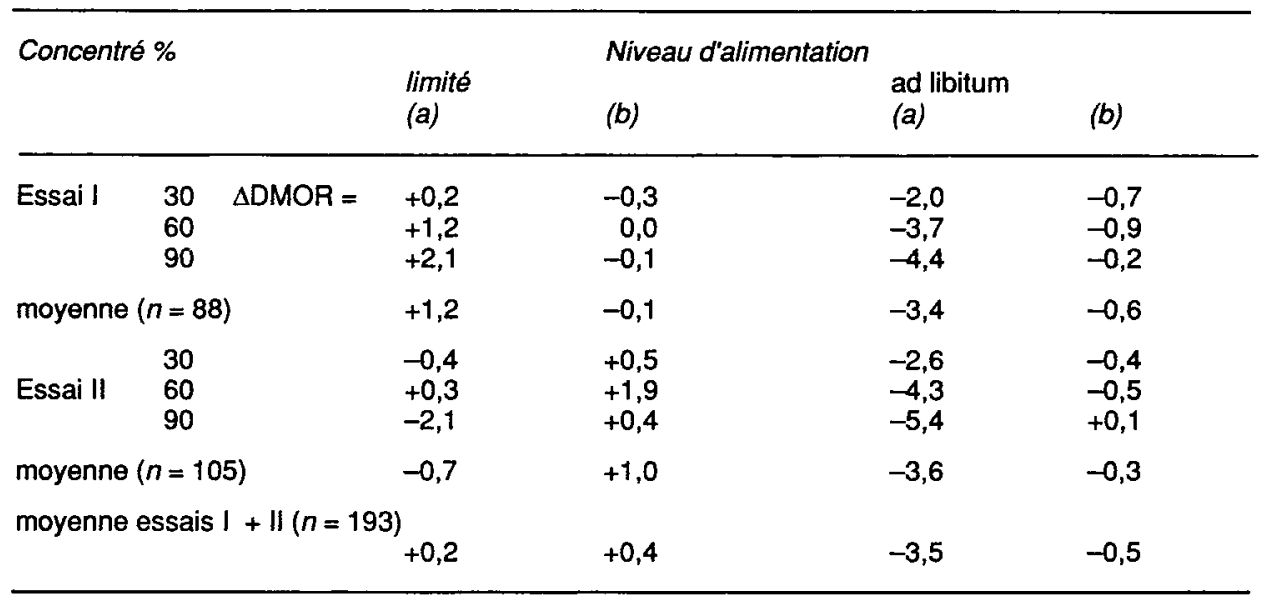

$\triangle D M O R=D M O R_{\text {mesurée }}-D M O R_{\text {calculée }}$

$D M O R_{\text {calculee }}=(1-\mathrm{C} / 100 D M O F+(C / 100) D M O C$

où $C$ : pourcentage de concentré dans la ration (base MS); DMOF : digestibilité mesurée du foin; $D M O C$ : digestibilité du concentré; (a) Tables INRA (1978); (b) DMOC calculée par régression (ct fig 2,3).

de digestibilité sont plus grands avec les concentrés riches en amidon qu'avec ceux riches en constituants pariétaux facilement digestibles (par ex la pulpe de betterave) et qu'ils diminuent quand la teneur en MAT de la ration augmente (Dulphy et al, 1987).

En conclusion, ce travail a montré que la digestibilité des aliments concentrés varie, tout comme celle des fourrages, avec le niveau alimentaire (autrement dit, avec la quantité totale d'aliments ingérés). Il importe donc, pour la prévision de la digestibilité d'une ration, que les digestibilités du fourrage et du concentré soient mesurées au même niveau alimentaire. À niveau alimentaire égal ou voisin de l'entretien, comme cela a été proposé par Van Es et Van der Meer (1980), les risques d'apparition de phénomènes d'interaction digestive, et les effets associatifs qui en découlent, sont faibles. D'ailleurs, un rapport du Rowett Research Institute (Wain- man et al, 1981), établi à partir de l'étude d'un ensemble de rations de composition variable, a confirmé que les effets associatifs de digestibilité étaient statistiquement néglibeables chez le mouton nourri à l'entretien. Minimiser les interactions digestives est d'autant plus justifié que l'on sait que le ruminant parvient à compenser les effets associatifs de digestibilité (baisse de la $D M O R$ ) par une diminution des pertes d'énergie sous forme de méthane et dans l'urine (Blaxter et Wainman, 1964) de sorte que les effets associatifs au niveau de l'énergie métabolisable sont plus faibles que ceux observés au niveau de la digestibilité (Vermorel et al, in INRA, 1978).

Malgré ces considérations, il n'est pas possible dans la pratique de moduler la digestibilité des aliments en fonction de leurs conditions d'emploi. On utilise pour les fourrages et les concentrés des valeurs fixes données par les tables (par ex : 
INRA, 1988). II faut ensuite introduire dans les calculs de ration des facteurs de correction tenant compte du niveau d'alimentation et de la valeur énergétique du fourrage (Vermorel et al, 1987). Les corrections sont parfois importantes et justifient par conséquent la prise en compte des interactions digestives.

L'importance prise par les effets du niveau alimentaire dans les phénomènes d'interaction digestive ne doit pas faire oublier celle du maintien dans le rumen de conditions favorables au déroulement normal de la digestion des aliments, en particulier celle de leurs constituants pariétaux. Dans les rations à forte proportion de concentré, ou du moins à teneur élevée en amidon, et/ou à teneur faible en MAT solubles, l'azote fermentescible peut rapidement devenir un facteur limitant de l'activité microbienne dans le rumen, et ladaptation des microorganismes à de tels régimes est, apparemment, longue (Latham et al, 1974; Mackie et al, 1978), en tous cas plus longue que la durée d'adaptation de 15 j que nous avons choisie dans nos essais.

\section{RÉFÉRENCES}

Balch CC, Campling RC (1962) Regulation of food intake in ruminants. Nutr Abstr Rev 32, 669-686

Berge P, Dulphy JP (1985) Étude des interactions entre fourrage et aliment concentré chez le mouton. I. Facteurs de variation du taux de substitution. Ann Zootech 34, 313334

Blaxter K, Wainman FW (1964) The utilization of the energy of different rations by sheep and cattle for maintenance and for fattening. $J$ Agric Sci 63, 113-128

Blaxter K, Wilson RS (1963) The assessment of a crop husbandry technique in terms of animal production. Anim Prod 5, 27-42

Dulphy JP, Andrieu JP, Rouel J (1987) Effet de la nature de l'aliment concentré sur les per- formances de vaches laitières recevant une ration à base de foin. Bull Tech CRZV Theix INRA 67, 43-47

Dulphy JP, Breton J, Louyot JM, Bienaime A (1983) Étude de la valeur alimentaire des pailles de céréales traitées ou non à la soude. III. Influence du niveau d'apport d'aliment concentré. Ann Zootech 32, 53-80

Dulphy JP, Kouassi A, Bienaime A (1982) Étude de la valeur alimentaire des pailles de céréales traitées ou non à la soude. II. Influence de la nature du complément énergétique. Ann Zootech 31, 215-232

Eadie JM, Hyldgaard-Jensen J, Mann SO, Reid RS, Whitelaw FG (1970) Observations on the microbiology and biochemistry of the rumen in cattle given different quantities of a pelleted barley straw. Br J Nutr 24, 157-177

Edionwe AO, Owen FG (1989) Relation of intake to digestibility of diets containing soyhulls and distillers dried grains. J Dairy Sci 72, 1786-1792

Giger S, Sauvant D (1983) Comparaison de différentes méthodes d'évaluation du coefficient d'utilisation digestive des aliments concentrés par le ruminant. Ann Zootech 32, 215246

Giger S, Sauvant D, Durand M, Hervieu J (1988) Influence de la nature de l'aliment concentré sur quelques paramètres de la digestion dans le rumen. Reprod Nutr Dev 28 , 117-118

Guerin H, Dulphy JP (1984) Influence de l'apport complémentaire de maïs, de pulpe de betterave ou de mélasse sur la valeur alimentaire d'un foin. Ann Zootech 33, 509-532

Hynd PI (1984) Effects of starch fermentation products on roughage digestion. J Agric Sci 103, 469-470

INRA (1978) Alimentation des Ruminants, INRA Publ, Versailles, $597 \mathrm{p}$

INRA (1988) Ruminant Nutrition. Recommended allowances and Feed Tables (R Jarrige, ed) John Libbey Eurotext Londres, Paris, $389 \mathrm{p}$

Joanning SW, Johnson DE, Barry BP (1981) Nutrient digestibility depressions in corn silagecorn grain mixtures fed to sieers. J Anim Sci 53, 1095-1103

Lamb CS, Eadie J (1979) The effect of barley supplements on the voluntary intake and digestion of low quality roughages by sheep. J Agric Sci Camb 92, 235-241 
Latham MJ, Sutton DJ, Sharpe ME (1974) Fermentation and micro-organisms in the rumen and the content of fat in the milk of cows given low roughage rations. J Dairy Sci 57, 803-810

Lyle LL, Johnson RR, Wilhite JV, Backus WR (1981) Ruminal characteristics in steers as affected by adaptation from forage to allconcentrate diets. J Anim Sci 53, 1383

Mackie RI, Gilchrist FMC, Robberts AM, Hannah PE, Schwartz HM (1978) Microbiological and chemical changes in the rumen during the stepwise adaptation of sheep to high concentrate diets. J Agric Sci 90, 241254

Mambrini M (1990) Étude du temps de séjour des résidus alimentaires dans le tube digestif des vaches laitières : aspects méthodologiques et facteurs de variation. Thèse de doctorat Université de Rennes I, $171 \mathrm{p}$

Michalet-Doreau B, Sauvant D (1989) Influence de la nature du concentré, céréales ou pulpe de betterave, sur la digestion chez les ruminants. INRA Prod Anim 2, 4, 235-244

Moe PW (1981) Energy metabolism of dairy cattle. J Dairy Sci 64, 1120-1139

Mould FL, Ørskov ER, Mann SO (1983a) Associative effects of mixed feeds. I. Effects of type and level of supplementation and the influence of the rumen fluid $\mathrm{pH}$ on the cellulolysis in vivo and dry matter digestion of various roughages. Anim Feed Sci Technol 10, 1530

Mould FL, Ørskov ER, Gauld SA (1983b) Associative effects of mixed feeds. II. The effects of dietary addition of bicarbonate salts on the voluntary intake and digestibility of diets containing various proportions of hay and barley. Anim Feed Sci Technol 10, 31-47

Muller A, Beranger C (1979) Utilisation des pulpes de betterave déshydratées en complément d'ensilage d'herbe par les bovins en croissance et a l'engrais. Bull Tech CRZV Theix, INRA 35, 53-58

Najar T, Giger S, Poncet C, Sauvant D (1988) Variations du temps de séjour du foin, du son de blé et de la pulpe de betteraves avec le niveau alimentaire et la proportion d'aliment concentré dans une ration mixte chez des chèvres laitières. Reprod Nutr Dév 28, 113-114

Orskov ER (1986) Starch digestion and utilization in ruminants. J Anim Sci 63, 1624-1633
Osbourn DF, Terry RA, Cammell SB, Outen GE (1970) Some aspects of feeding supplements of Inaize meal and sodium bicarbonate upon the digesta of forage cellulose by sheep. Proc Nutr Soc 29, 12A-13A

Peyraud JL, Mambrini M, Rulquin H (1988) Temps de séjour comparé de l'ensilage de maîs égrené et d'un aliment concentré dans une ration mixte distribuée à deux niveaux d'ingestion à des vaches laitières. Reprod Nutr Dév 28(1), 111-112

Poncet C, Gomez L, Michalet-Doreau B, Geay Y (1987a) Temps de séjour comparé du foin, du son de blé et de la pulpe de betterave, d'une ration mixte distribuée à deux niveaux d'alimentation à des moutons et des taurillons. Reprod Nutr Dév 27(1B), 219-220

Poncet C, Gonzalez J, Michalet-Doreau B (1987b) Effets du pourcentage de concentré de la ration et du niveau d'ingestion sur la vitesse de passage dans le rumen de différents types d'aliments chez le mouton. $R e$ prod Nutr Dév 27 (1B), 257-258

Robinson PN, Tamminga $S$, Van Vuuren AM (1987) Influence of declining level of feed intake and varying the proportion of starch in the concentrate on rumen ingesta quantity, composition and kinetics of ingesta turnover in dairy cows. Livest Prod Sci 17, 37-62

Seebeck RM (1973) The effect of body-weight loss on the composition of Brahman cross and Africander cross steers. I. Empty body weight, dressed carcass weight and offal components. J Agric Sci 80, 201-210

Shriver BJ, Sargent JP, Crawford RJ, Thayne WV, Hoover WH (1984) Digesta flow and pH effects on microbial metabolism. $J$ Anim Sci 59 (suppl 1), 419 (abstr)

Van Es AJH, Van der Meer JM (1980) Chemical analysis for the prediction of nutritive value. 31 st Annu Meet EAAP 1-4 sept 1980, Munich, 48 p

Vérité R, Dulphy JP (1981) Effet de la nature, de l'aliment concentré sur l'ingestion et les performances des vaches laitières. Bull Tech CRZV Theix, 45, 15-21

Vermorel M, Coulon JB, Journet M (1987) Révision du système des unités fourragères (UF). Bull Tech CRZV Theix 70, 9-18

Wainman FW, Dewey PJS, Boyne AW (1981) Compound Feedingstuffs for Ruminants. Rowett Res Inst - Feedingstuffs Evaluation Unit, 3rd Report 